\title{
IRREDUCIBILITY IN ALGEBRAIC GROUPS AND REGULAR UNIPOTENT ELEMENTS
}

\author{
DONNA TESTERMAN AND ALEXANDRE ZALESSKI
}

(Communicated by Pham Huu Tiep)

\begin{abstract}
We study (connected) reductive subgroups $G$ of a reductive algebraic group $H$, where $G$ contains a regular unipotent element of $H$. The main result states that $G$ cannot lie in a proper parabolic subgroup of $H$. This result is new even in the classical case $H=\mathrm{SL}(n, F)$, the special linear group over an algebraically closed field, where a regular unipotent element is one whose Jordan normal form consists of a single block. In previous work, Saxl and Seitz (1997) determined the maximal closed positive-dimensional (not necessarily connected) subgroups of simple algebraic groups containing regular unipotent elements. Combining their work with our main result, we classify all reductive subgroups of a simple algebraic group $H$ which contain a regular unipotent element.
\end{abstract}

\section{INTRODUCTION}

Let $H$ be a reductive linear algebraic group defined over an algebraically closed field $F$. Throughout this text 'reductive' will mean 'connected reductive'. A unipotent element $u \in H$ is said to be regular if the dimension of its centralizer $C_{H}(u)$ coincides with the rank of $H$ (or, equivalently, $u$ is contained in a unique Borel subgroup of $H$ ). Regular unipotent elements of a reductive algebraic group exist in all characteristics (see [22]) and form a single conjugacy class. These play an important role in the general theory of algebraic groups. In this paper we study reductive subgroups of $H$ containing a regular unipotent element of $H$. We find that such subgroups are irreducible in the sense of Serre [17, who has generalized the common notions of irreducible, completely reducible, and reducible linear groups as follows.

Definition 1.1. Let $H$ be a reductive linear algebraic group. A subgroup $G$ of $H$ is called $H$-irreducible if $G$ is contained in no proper parabolic subgroup, $H$ completely reducible (hereafter referred to as $H$-cr) if, whenever $G$ belongs to a parabolic subgroup $P$ of $H$, then $G$ belongs to a Levi subgroup of $P$, and $H$ reducible if $G$ lies in a proper parabolic subgroup of $H$.

The main result of this paper states that for $G, H$ reductive groups, if $G$ is a closed subgroup of $H$ containing a regular unipotent element of $H$, then $G$ is $H$-irreducible. This result is new even for the classical situation where $H$ is the general linear group. Closed subgroups of simple algebraic groups containing a regular unipotent element were studied, and maximal positive-dimensional such

Received by the editors April 23, 2011.

2010 Mathematics Subject Classification. Primary 20G05, $20 \mathrm{G} 07$. 
subgroups were classified by Saxl and Seitz in 16; however, they did not treat the irreducibility phenomenon that is the subject of this paper.

The set of regular unipotent elements is a dense open set in the variety of unipotent elements of $H$. In a sense they are the 'largest' unipotent elements, while the nontrivial elements of root groups are the 'smallest'. In many situations, it is useful to know the subgroups containing elements of this or other special kinds. (See Saxl's survey [15] for an overview and bibliography.)

The problem of determining closed subgroups of simple algebraic groups $H$ containing a regular unipotent element has already attracted considerable attention. In [23], I. Suprunenko determined closed irreducible semisimple subgroups of $\operatorname{GL}(n, F)$ containing a regular unipotent element. When $\operatorname{char}(F)$ is 0 or a large enough prime, a regular unipotent element lies in a closed subgroup of $H$ isomorphic to (P)SL $(2, F)$. A primary problem solved in [25, 14 was to classify all situations when a unipotent element is contained in a closed subgroup of type $A_{1}$; the case of regular unipotent elements was crucial and in some sense the most difficult. Properties of the centralizer $C$ of a regular unipotent element and of its normalizer $N_{G}(C)$ were investigated in [13. The most extensive study of the overgroups of regular unipotent elements to date was carried out by Saxl and Seitz in [16], where they determined maximal (not necessarily connected) positive-dimensional subgroups of $H$ containing a regular unipotent element. As a maximal positive-dimensional subgroup is either the normalizer of a reductive subgroup or is a parabolic subgroup (by the Borel-Tits theorem [2]), and as each parabolic subgroup contains a representative of every unipotent class (and so in particular contains a regular unipotent element), their article is concerned with determining reductive maximal subgroups containing a regular unipotent element.

As an application of our main result, we are able to deduce from [16, Theorems A and B] the classification of semisimple subgroups $G$ of simple algebraic groups $H$ such that $G$ contains a regular unipotent element of $H$. (See Theorem 1.4 below.) Indeed, given such a subgroup $G \subset H$, one embeds $G$ in a maximal positive-dimensional subgroup, which is one of the groups given by the results of [16. If $M^{\circ}$ is reductive, then $G$ lies in the semisimple group $\left[M^{\circ}, M^{\circ}\right]$ and we proceed inductively. Otherwise, $M$ is a parabolic subgroup and one is faced with the question of whether $G$ lies in a Levi factor of $M$ in order to again argue inductively. Our main result (Theorem 1.2) solves this problem by showing that $G$ cannot lie in a proper parabolic subgroup of $H$.

Theorem 1.2. Let $G$ be a reductive subgroup of the reductive group $H$ containing a regular unipotent element of $H$. Then $G$ is not contained in any proper parabolic subgroup of $H$. In other words, $G$ is $H$-irreducible.

The special case $H=\mathrm{SL}(n, F)$ seems worth stating explicitly:

Corollary 1.3. Let $G \subset \mathrm{GL}(n, F)$ be a reductive linear algebraic group. Suppose that $G$ contains an element whose Jordan normal form consists of a single block. Then $G$ is irreducible.

We first note that the theorem is clearly true if $\operatorname{char}(F)=0$, since all $F G$ modules are then completely reducible, and so if $G$ lies in a parabolic subgroup, it necessarily lies in a Levi factor of this group.

Observe that one cannot drop the hypothesis that $G$ is connected. Indeed, a similar statement for finite reductive groups is false: there exists a reducible 
TABle 1. Semisimple subgroups $G \subset H$ containing a regular unipotent element

\begin{tabular}{|l|l|}
\hline$H$ & $G$ \\
\hline$A_{6}$ & $G_{2}, p \neq 2$ \\
$A_{5}$ & $G_{2}, p=2$ \\
\hline$C_{3}$ & $G_{2}, p=2$ \\
\hline$B_{3}$ & $G_{2}, p \neq 2$ \\
\hline$D_{4}$ & $G_{2}, p \neq 2$ \\
& $B_{3}$ \\
\hline$E_{6}$ & $F_{4}$ \\
\hline$A_{n-1}, n>1$ & $C_{n / 2}, n$ even \\
& $B_{(n-1) / 2}, n$ odd,$p \neq 2$ \\
\hline$D_{n}, n>4$ & $B_{n-1}$ \\
\hline
\end{tabular}

representation $\rho: \operatorname{PSL}(2, p) \rightarrow \operatorname{SL}(p, F)$, where $F$ is of characteristic $p>0$ such that the image of $\rho$ contains a unipotent element with a single Jordan block matrix. (See, for example, [4, p. 590].)

As mentioned above, we will apply the above results and the main result of 16 to obtain the following classification of semisimple subgroups of simple groups $H$ containing a regular unipotent element.

Theorem 1.4. Let $G$ be a closed semisimple subgroup of the simple algebraic group $H$, containing a regular unipotent element of $H$. Then either the pair $H, G$ is as given in Table 1 or $G$ is isomorphic to $(\mathrm{P}) \operatorname{SL}(2, F)$ and $p=0$ or $p>h$, where $h$ is the Coxeter number for $H$. Moreover, for each pair of root systems $\left(\Phi_{H}, \Phi_{G}\right)$ as in the table, respectively, for $\left(\Phi_{H}, A_{1}, p\right)$, with $p=0$ or $p>h$, there exists a closed simple subgroup $X \subset H$ of type $\Phi_{G}$, respectively $A_{1}$, containing a regular unipotent element of $H$.

The conjugacy classes of such subgroups can be deduced from the known structure of maximal connected subgroups of $H$ (see [19] and [11]).

Our methods for proving the main theorem differ according to whether $H$ is of classical or of exceptional type. In the former case we use results on indecomposable representations of simple algebraic groups applied to our group $G$ and the natural $H$-module. These include general results such as Lemma 2.2, as well as more special results on splitting certain $G$-modules of composition length 2 obtained by McNinch [12]. For the exceptional groups $H$, we use the classification results on maximal subgroups and subgroups of type $A_{1}$ obtained by Seitz, Liebeck and Testerman 11, 25.

It is well-known that the Jordan normal form of the Kronecker product of two unipotent Jordan blocks is not similar to a Jordan block matrix. It is probably worth mentioning the following generalization of this fact to arbitrary simple algebraic groups: if $X$ and $Y$ are nonabelian commuting reductive subgroups of a simple algebraic group $H$, then the product $X Y$ contains no regular unipotent element of $H$. This is a special case of Proposition 2.3.

Notation and conventions. We will write $[X, X]$, or $X^{\prime}$, for the derived subgroup of a group $X$. The order of an element $x \in X$ is denoted by $|x|$. We use $C_{X}(M)$ to denote the centralizer in $X$ of a subset $M \subset X$. 
Below $F$ is an algebraically closed field of characteristic $p \geq 0$. The term 'a simple algebraic group' designates (unless otherwise stated) a simply connected simple algebraic group defined over $F$. To simplify the language we often write $G=A_{n}, B_{n}$, etc., instead of the more precise ' $G$ is a simple simply connected linear algebraic group of type $A_{n}, B_{n}$,' etc. If $G$ is an algebraic group, then $G^{\circ}$ is its connected component. If $G$ is reductive, then $[G, G]$ coincides with the subgroup generated by the unipotent elements of $G$. All $F G$-modules under consideration are rational.

As usual, $G$ determines its root system $\Phi$ and the weight lattice $\Omega(G)$. We fix a Borel subgroup $B$ of $G$ and a maximal torus $T \subset B$. We denote the corresponding set of simple roots by $\Delta$ and the positive roots by $\Phi^{+}$; dominant weights are denoted by $\Omega^{+}(G)$. We label Dynkin diagrams as in 3 . and let $\Delta=\left\{\alpha_{1}, \ldots, \alpha_{\ell}\right\}$, with associated fundamental dominant weights $\omega_{1}, \ldots, \omega_{\ell}$. The 1-dimensional connected unipotent group normalized by $T$, with action given by the character $\alpha \in \Phi$, is denoted by $U_{\alpha}$ and its elements by $x_{\alpha}(t)(t \in F)$. For $\mu \in \Omega^{+}(G)$, we let $V(\mu)$ denote the irreducible $F G$-module of highest weight $\mu$, and $W(\mu)$ the indecomposable Weyl module of highest weight $\mu$. By a 'classical group', we mean a simple simply connected algebraic group of type $A_{n}(n \geq 1), B_{n}(n>2), C_{n}(n>1)$ or $D_{n}$ $(n>3)$. Except when $G=B_{n}$ and $p=2$, we take the so-called 'natural' module for $G$ to be the irreducible module with highest weight $\omega_{1}$. In the exceptional case, the natural module for $G=B_{n}$ is the $(2 n+1)$-dimensional reducible $F G$-module equipped with a nondegenerate quadratic form, whose associated bilinear form has a 1-dimensional radical.

\section{LiNEAR REPRESENTATIONS}

Unless stated otherwise, we assume throughout this section that $H$ is a simple algebraic group. We first recall some results from the representation theory of simple algebraic groups and establish a result (Proposition 2.3) which will reduce the problem to the study of simple subgroups. In addition, we will prove Theorem 1.2 in the case where $H$ is the group $\operatorname{SL}(n, F)$.

Lemma 2.1. Let $G \subset H$ be reductive algebraic groups, and let $u \in G$ be unipotent. If $u$ is regular in $H$, then so are all regular unipotent elements of $G$.

Proof. Let $x$ be a regular unipotent element in $G$. Then $u$ lies in the closure of the $G$-class of $x$, and this of course lies in the closure of the $H$-class of $x$. If $x$ is not regular in $H$, this class has dimension strictly less than the dimension of the $H$-class of $u$, which is a contradiction. Therefore $x$ is a regular element in $H$, as claimed.

We require some additional notation; let $\Phi(H)$ denote the root system of $H$ and, for $\alpha \in \Phi(H), U_{\alpha}$ denotes the corresponding root subgroup with respect to a fixed maximal torus $T_{H}$ of $H$. Fix a base $\Delta(H) \subset \Phi(H)$ and let $\Phi^{+}(H)$ denote the corresponding set of positive roots, $B_{H} \supset T_{H}$ the corresponding Borel subgroup of $H$, with $R_{u}\left(B_{H}\right)=U$ and $U_{1}$ the subgroup $\Pi_{\beta \in \Phi^{+}(H) \backslash \Delta(H)} U_{\beta}$. Finally, we also recall that the centralizer of a regular unipotent element contains no noncentral semisimple elements; see [20, Ch. III, 1.14(a)].

We will need the following standard result from the literature; see e.g. Humphreys [7, 12.4]: 
Lemma 2.2. Let $E$ be an indecomposable module for a simple algebraic group of composition length 2. Let $\mu, \lambda$ be the highest weights of $E / L, L$, resp., where $L$ is the maximal submodule of $E$. Then either $\lambda>\mu$ or $\mu>\lambda$, and in the latter case $E$ is isomorphic to $W(\mu) / M$, where $M$ is a submodule of $W(\mu)$.

Actually we get that $W(\mu)$ is reducible if there exists an indecomposable module $E$ as in the above lemma with $\mu>\lambda$.

Proposition 2.3. Let $H$ be a simple algebraic group and let $X, Y \subset H$ be proper subgroups such that $[X, Y]=1$ and such that $X$ and $Y$ each contain a semisimple element of $H \backslash Z(H)$. Then $X Y$ contains no regular unipotent elements of $H$.

We begin with the following:

Lemma 2.4. Let $H$ be a simple algebraic group and $u, u^{\prime} \in U=R_{u}\left(B_{H}\right)$. Suppose that $u$ is regular and $u u^{\prime}=u^{\prime} u$. Then either $u^{\prime}$ is regular or $u^{\prime} \in U_{1}$.

Proof. Note first that the lemma is true for groups of rank 2. For this, observe that $A_{2}, B_{2} \cong C_{2}, G_{2}$ are the only groups in question. In the first case verifying the lemma is a matter of elementary matrix computations. If $G$ is of type $B_{2}=C_{2}$, the explicit commutator formula for $\left[u, u_{1}\right]$ is available in [21, Lemma 33], and for $G$ of type $G_{2}$ a similar formula is written down in [5, p. 192]. Using these, one easily arrives at the conclusion. (One can also use the commutator formulas for the root subgroups in [6, Theorem 1.12.1].)

In general, express $u, u^{\prime}$ as follows: $u=\prod_{\alpha \in \Delta(H)} x_{\alpha}\left(t_{\alpha}\right) \cdot \prod_{\beta \in \Phi^{+}(H) \backslash \Delta(H)} x_{\beta}\left(t_{\beta}\right)$, and $u^{\prime}=\prod_{\alpha \in \Delta(H)} x_{\alpha}\left(t_{\alpha}^{\prime}\right) \cdot \prod_{\beta \in \Phi^{+}(H) \backslash \Delta(H)} x_{\beta}\left(t_{\beta}^{\prime}\right)$, where $t_{\alpha}, t_{\beta}, t_{\alpha}^{\prime}, t_{\beta}^{\prime} \in F$.

Suppose the contrary; that is, $u^{\prime}$ is not regular and $u^{\prime} \notin U_{1}$. By [20. Ch. III, 1.13] $u^{\prime}$ is regular if and only if $t_{\alpha}^{\prime} \neq 0$ for all $\alpha \in \Delta(H)$. Therefore, there is $\beta \in \Delta(H)$ such that $t_{\beta}^{\prime}=0$. Moreover, as $u^{\prime} \notin U_{1}$, there exists a pair of roots $\delta, \gamma \in \Delta(H)$ adjacent to each other in the Dynkin diagram of $H$ and such that $t_{\gamma}^{\prime}=0, t_{\delta}^{\prime} \neq 0$. Let $P=\left\langle B, U_{-\gamma}, U_{-\delta}\right\rangle$. Then $P$ is a parabolic subgroup of $H$. Let $L$ be its standard Levi subgroup with $L^{\prime}:=\left\langle U_{ \pm \gamma}, U_{ \pm \delta}\right\rangle$. Let $h: P \rightarrow L$ be the natural surjection. Set $\bar{u}=h(u), \bar{u}^{\prime}=h\left(u^{\prime}\right)$. Then $\bar{u}, \bar{u}^{\prime} \in L^{\prime}$ and $\overline{u u}^{\prime}=\bar{u}^{\prime} \bar{u}$. Let $\Phi_{L}, \Delta_{L}, \Phi_{L}^{+}$be the root system, simple root system and the set of positive roots for $L^{\prime}$, with respect to the maximal torus $T_{H} \cap L$ and Borel subgroup $B_{H} \cap L$. Then $\Delta_{L}=\{\gamma, \delta\}$ and

$$
\bar{u}=x_{\gamma}\left(t_{\gamma}\right) x_{\delta}\left(t_{\delta}\right) \cdot \prod_{\beta \in\left(\Phi_{L}^{+} \backslash \Delta_{L}\right)} x_{\beta}\left(t_{\beta}\right)
$$

is regular in $L^{\prime}$ and

$$
\overline{u^{\prime}}=x_{\delta}\left(t_{\delta}^{\prime}\right) \cdot \prod_{\beta \in \Phi_{L}^{+} \backslash \Delta_{L}} x_{\beta}\left(t_{\beta}^{\prime}\right)
$$

is not regular. In addition, $\overline{u u}^{\prime}=\bar{u}^{\prime} \bar{u}$. This is a contradiction, as the result holds in the rank two group $L^{\prime}$.

Proof of Proposition 2.3. Suppose the contrary, and let $u \in X Y$ be a regular unipotent element. Then $u=u_{1} u_{2}=u_{2} u_{1}$ for some unipotent elements $u_{1} \in$ $X, u_{2} \in Y$. Moreover, neither of $u_{1}, u_{2}$ is regular, as each of $X, Y$ contains a noncentral semisimple element. As $u u_{i}=u_{i} u$ for $i=1,2$, Lemma 2.3 implies that $u_{1}, u_{2} \in U_{1}$. Then $u=u_{1} u_{2} \in U_{1}$, which is false by [20, Ch. III, 1.13]. 
The above proof shows slightly more: a regular element $u$ has no factorization $u=u_{1} u_{2}$, where $C_{H}\left(u_{i}\right)$ contains a semisimple element of $H \backslash Z(H)$.

Theorem 1.2 will follow directly from the following result and Proposition 2.3 for the case $H$ a simple algebraic group.

Theorem 2.5. Let $G \subset H$ be a simple closed subgroup of the simple algebraic group $H$, and suppose $G$ contains a regular unipotent element of $H$. Then $G$ is $H$-irreducible; that is, $G$ is not contained in any parabolic subgroup of $H$.

We first establish an elementary lemma.

Lemma 2.6. Let $P \subset H$ be a proper parabolic subgroup, properly containing a Borel subgroup of $H$. Let $L \subset P$ be a Levi subgroup and $\pi: P \rightarrow L$ the natural surjection. Then for $u \in P$ a regular unipotent element of $H, u \notin L$ and $\pi(u)$ is a regular unipotent element of $L$.

Proof. Let $T_{H} \subset B_{H}$ be as above. We can assume that $B_{H} \subset P$. Then $L=$ $\left\langle T_{H}, U_{ \pm \alpha}: \alpha \in J\right\rangle$ for some proper nonempty subset $J$ of $\Delta(H)$. Using the notation of the proof of Lemma 2.4, we have $u=\prod_{\alpha \in \Delta(H)} x_{\alpha}\left(t_{\alpha}\right) \cdot \prod_{\beta \in \Phi^{+}(H) \backslash \Delta(H)} x_{\beta}\left(t_{\beta}\right)$. By [20, Ch. III, 1.13] $u$ regular implies $t_{\alpha} \neq 0$ for all $\alpha \in \Delta(H)$. This implies the first assertion, as if $u \in L$, then $t_{\alpha}=0$ whenever $\alpha \notin J$. The second assertion also follows since $\pi(u)=\prod_{\alpha \in J} x_{\alpha}\left(t_{\alpha}\right) \cdot \prod_{\beta \in \Phi_{L}^{+} \backslash J} x_{\beta}\left(t_{\beta}\right)$, where $\Phi_{L}^{+}$are the roots in $\Phi^{+}(H)$ which are linear combinations of the roots in $J$.

Lemma 2.7. Assume $\operatorname{char}(F)=0$ and let $G \subset H$ be a reductive subgroup of the simple algebraic group $H$ such that $u \in G$ is a regular unipotent element of $H$. Then $G$ is $H$-irreducible

Proof. This follows directly from Lemma 2.6 and the fact that all $F G$-modules are completely reducible. We apply this to the $F G$-modules induced by the action of $G$ on the unipotent radical of any parabolic subgroup containing $G$ to see that $G$ lies in a Levi factor, contradicting Lemma 2.6.

In Lemmas 2.8 and 2.9 below we consider two special cases for $p=2$. The claim (2) of Lemma 2.8 is stated without proof in [16, p. 373]. We provide a proof here for the sake of completeness. Lemma 2.9 would follow from Lemma 2.8 as soon as one shows that every indecomposable representation of $G=G_{2}$ of degree 7 in characteristic 2 preserves a quadratic form. However, it seems simpler to argue directly.

Lemma 2.8. Let $G=B_{n}$ and $p=2$. Let $\phi: G \rightarrow H=A_{2 n}$ be an indecomposable representation.

(1) The composition factors of $\phi$ are of dimensions 1 and $2 n$. If the socle of the FG-module corresponding to $\phi$ is one-dimensional, then $\phi(G)$ stabilizes a nondegenerate quadratic form on $F^{2 n+1}$ and hence $\phi(G)=\operatorname{SO}(2 n+1, F) \subset H=$ $\mathrm{SL}(2 n+1, F)$.

(2) $\mathrm{SO}(2 n+1, F)$, and hence $\phi(G)$, contains no regular unipotent element of $H$, equivalently, no matrix similar to $J_{2 n+1}$, the unipotent Jordan block of size $2 n+1$. 
Proof. (1) It is well-known that the minimal dimension of a nontrivial $F G$-module is $2 n$ with highest weight $\mu=2^{k} \omega_{1}$ and that there is no $(2 n+1)$-dimensional irreducible $F G$-module. Therefore, the composition length of $G$ on the natural module $V$ for $H$ is 2 . Let $\mu$ be the highest weight of the nontrivial $F G$-composition factor of $V$. By [9, II.12.9, II.10.17(2)], the number of nonequivalent nonsplit extensions of $V_{0}$ by $V_{2^{k} \omega_{1}}$ is equal to the number of nonequivalent nonsplit extensions of $V_{0}$ by $V_{\omega_{1}}$. So every nonsplit extension of $V_{0}$ by $V_{2^{k} \omega_{1}}$ can be obtained by a Frobenius twist from a nonsplit extension of $V_{0}$ by $V_{\omega_{1}}$. Therefore, it suffices to deal with $\mu=\omega_{1}$. Replacing if necessary $V$ by its dual, by Lemma 2.2 , we deduce that $V$ is a quotient of $W\left(\omega_{1}\right)$, the Weyl module with highest weight $\omega_{1}$, whose dimension is $2 n+1$. So $V \cong W\left(\omega_{1}\right)$. Furthermore, there is an indecomposable $F G$-module of dimension $2 n+1$ with quadratic form $Q$ defining the orthogonal group $\mathrm{O}(2 n+1, F)$, and this module fixes a nonzero vector, as per our earlier discussion concerning the natural $B_{n}$-module. The group $\mathrm{SO}(2 n+1, F)$ is known to be of type $B_{n}$; see [1. Sections 23.4 and 23.5]. By the above remarks, this representation is also a twist of the Weyl module $W\left(\omega_{1}\right)$. As $\mathrm{O}(2 n+1, F)$ is stable under the Frobenius endomorphism, it follows that $\phi(G)$ coincides with $\mathrm{SO}(2 n+1, F)$. This implies (1).

(2) Suppose the contrary, and let $u \in G$ be a unipotent element having one Jordan block in its action on $V$, so $V$ is uniserial as an $F\langle u\rangle$-module. So the socle of the $F G$-module $V$ is 1-dimensional and hence $\phi(G)$ preserves a quadratic form on $V$ by (1). Then $u$ stabilizes a totally singular subspace $W$ of dimension $n-1$. Then $\operatorname{Stab}_{G}(W)$ is a parabolic subgroup $P$ of $G$, and $u \in P$. Then $X:=W^{\perp} / W$ is a vector space of dimension 3, and the quadratic form $Q$ induces on $X$ a nondegenerate quadratic form defining therefore an orthogonal group $\mathrm{O}(3, F)$. As both $W, W^{\perp}$ are $u$-stable, and $V$ is uniserial for $u$, so is $X$. Therefore, $\operatorname{SO}(3, F)$ contains a uniserial element $u^{\prime}$, say, which is the projection of $u$. Obviously, the order of $u^{\prime}$ is 4 . However, this is false as $\mathrm{SO}(3, F) \cong \mathrm{SL}(2, F)$, so all unipotent elements of $\mathrm{SL}(2, F)$ are of order 2 .

Lemma 2.9. Let $p=2$ and $G=G_{2}$. Suppose that $G \subset H=\operatorname{SL}(7, F)$. Then $G$ contains no regular unipotent element of $H$.

Proof. It is well-known that the minimal dimension of a nontrivial $F G$-module is 6 with highest weight $\mu=2^{k} \omega_{1}$, and that there is no 7-dimensional irreducible $F G$ module. Therefore, the composition length of $G$ on the natural module $V$ for $H$ is 2 . Let $\mu$ be the highest weight of the nontrivial $F G$-composition factor of $V$. Arguing as in the proof of the previous lemma, applying again [9, II.12.9, II.10.17(2)] and Lemma 2.2, we see that we may assume that $V$ is a quotient of the Weyl module of highest weight $\mu=\omega_{1}$; but this latter is of dimension 7 , so we have that $V$ is isomorphic to the Weyl module of highest weight $\omega_{1}$.

We now apply a result of 24] which describes the action of the fundamental root group elements of $G=G_{2}$ on $W\left(\omega_{1}\right)$. Let $E_{i j} \in \mathrm{GL}(7, F)$ denote the matrix with 1 at the position $(i, j)$ and zero elsewhere. As $p=2$, 24, the proof of Theorem 3.0, p. 43] shows that the matrix of $x_{\alpha_{1}}(1) x_{\alpha_{2}}(1)$ (a regular unipotent element in $G$ ) with respect to a fixed basis of $W\left(\omega_{1}\right)$ is $\left(1+E_{12}+E_{45}+E_{67}+E_{35}\right)\left(1+E_{23}+E_{56}\right)=$ $1+E_{12}+E_{45}+E_{67}+E_{35}+E_{23}+E_{56}+E_{13}+E_{46}+E_{36}$. This is not a regular unipotent element in $\operatorname{SL}(7, F)$ as the term $E_{34}$ does not occur in this expression. Since regular unipotent elements of $G_{2}$ form a single $G_{2}$-conjugacy class, the result follows from Lemma 2.1 
The following lemma is the result [23, 1.9], which is crucial in our analysis.

Lemma 2.10. Let $\phi: G \rightarrow H=\operatorname{SL}(n, F), n>1$, be a nontrivial irreducible representation of the simple algebraic group $G$, with highest weight $\lambda=a_{1} \omega_{1}+\cdots+$ $a_{\ell} \omega_{\ell}$. Suppose that $\phi(G)$ contains a regular unipotent element of $H$. Then one of the following holds (where $k \geq 0$ is an integer and $k=0$ if $p=0$ ):

(i) $G=A_{1}, \lambda=p^{k} m \omega_{1}$ and $n=m+1 \leq p$ if $p>0$;

(ii) $G=A_{\ell}, \ell>1, \lambda=p^{k} \omega_{1}$ or $p^{k} \omega_{\ell}$ and $n=\ell+1$;

(iii) $G=C_{\ell}, \ell>2, \lambda=p^{k} \omega_{1}$ and $n=2 \ell$;

(iv) $G=C_{2}, \lambda=p^{k} \omega_{1}$ and $n=4$, or $\lambda=p^{k} \omega_{2}$ and $n=5$ for $p \neq 2$ and $n=4$ otherwise;

(v) $G=B_{\ell}, \ell>2, \lambda=p^{k} \omega_{1}$ and $n=2 \ell+1$ for $p \neq 2$ and $n=2 \ell$ otherwise;

(vi) $G=G_{2}, p \neq 3, \lambda=p^{k} \omega_{1}$, and $n=7$ if $p \neq 2$ and 6 otherwise;

(vii) $G=G_{2}, p=3$ and $\lambda=p^{k} \omega_{1}$ or $p^{k} \omega_{2}$ and $n=7$.

Proposition 2.11. Theorem 2.5 is true for $H=\mathrm{SL}(n, F)$.

Proof. Suppose the contrary, that is, that $G$ is $H$-reducible, so $G$ acts reducibly on the natural $F H$-module $V$. Let $u \in G$ be a unipotent element that is regular in $H$. This is equivalent to saying that $\operatorname{dim} V^{u}=1$, where $V^{u}$ is the fixed point subspace of $u$ on $V$. It follows that every $F G$-submodule of $V$ is indecomposable. Let $0=V_{0} \subset V_{1} \subset \cdots \subset V_{t}=V$ be a composition series for the $F G$-module $V$; we have $t>1$. Then $\left.u\right|_{V_{2}}$ is a regular unipotent element in $\operatorname{SL}\left(V_{2}\right)$. Note that as $V_{2}$ is indecomposable and $\left.u\right|_{V_{1}}$ and $\left.u\right|_{V_{2} / V_{1}}$ are regular elements, we may assume that the composition factors have highest weights as specified in Lemma 2.10.

We will apply the results of Jantzen [8] and McNinch [12. One of them asserts that any $F G$-module of dimension $m$ is completely reducible if $m \leq p \cdot \ell$, where $\ell$ is the rank of $G$ (see [8, Theorem A] for the case $G=A_{1}$ and [12, Corollary 1.1.1] for the general case). We first note that for $G=A_{1}$, since $u$ has order $p$, the dimension of $V_{2}$ is at most $p$. In particular, the above criterion shows that $V_{2}$ is completely reducible, contradicting our assumptions. Now we turn to the other representations of Lemma 2.10. We have $\operatorname{dim} V_{2} \leq 2(\ell+1)$, respectively $4 \ell, 10,2(2 \ell+1)$, for $G$ as in (ii), respectively (iii), (iv), (v) of Lemma 2.10, Again applying the criterion of [12] and recalling that $V_{2}$ is indecomposable, we reduce to the following configurations:

(a) $G=A_{\ell}, \ell>1$ and $p=2$;

(b) $G=C_{\ell}$ and $p \leq 3$;

(c) $G=B_{\ell}$ and $p \leq 3$;

(d) $G=G_{2}$.

For the cases (a) - (d), we use a stronger result [12, Theorem 1], which asserts that an $F G$-module $W$ of dimension at most $p \cdot C$ is completely reducible (where $C=$ $\ell(\ell+1) / 2$ for $G$ of type $A_{\ell}, \ell(\ell-1)$ for types $B_{\ell}, C_{\ell}$ and 3 for type $\left.G_{2}\right)$, unless the highest weights of the composition factors of $W$ occur in [12, Table 5.1.1]. Applying this to the cases (a) - (d), we obtain a contradiction to the indecomposability of $V_{2}$ unless either $p=2, G \cong B_{\ell}$ or $C_{\ell}$ and the highest weights of the composition factors are 0 and $2^{k} \omega_{1}$, or $G \in\left\{C_{2}, G_{2}\right\}$ and $p \leq 3$. In the first case, note that there is a surjective homomorphism $B_{\ell} \rightarrow C_{\ell}$ when $p=2$, and the highest weight of the irreducible $B_{\ell}$-module induced by the irreducible $C_{\ell}$-module of highest weight 
$\omega_{1}$ is $\omega_{1}$ as well. Thus it suffices to consider only the $B_{\ell}$ case. Thus $V_{2}$ is an indecomposable $B_{\ell}$-module of composition length 2 with factors of highest weights $2^{k} \omega_{1}$ and 0 . By Lemma 2.8, the image of $B_{\ell}$ in $H$ (and hence the subgroup $C_{\ell} \subset H$ ) contains no regular element of $H$.

Let $G=C_{2}$. Consider first the case where $p=2$. Then $|u|=4$, and hence $\operatorname{dim} V_{2} \leq|u|=4$. But there exists no reducible nontrivial $F G$-module of dimension 4 , so $p>2$. Now let $p=3$. Note that the central involution of $C_{2}$ is nontrivial in any irreducible representation of dimension 4. It follows that either both composition factors of $V_{2}$ are of dimension 4 or $V_{2}$ has no composition factor of dimension 4. In the latter case, $\operatorname{dim} V_{2} \leq 6$, and applying again 12 we get a contradiction. Suppose that $\operatorname{dim} V_{2}=8$. By Lemma 2.10 the highest weights of the composition factors of $V_{2}$ are $\mu:=3^{k} \omega_{1}$ and $\lambda:=3^{m} \omega_{1}$, and we may assume $k \leq m$. By [12, Lemma 2.3.3(b)], we can assume that $k=0$, and $m \geq 1$ by [12, Lemma 2.3.1(b)]. Set $\nu=\lambda-\mu=(q-1) \omega_{1}$, for $q=3^{m}$. We now normalize the inner product on $\mathbf{Z} \Phi$ so that for $\alpha \in \Phi$ a long root, we have $(\alpha, \alpha)=1$. Then we will apply [18, (6.2)], which shows that $2\left(\lambda+\omega_{1}+\omega_{2}, \nu\right)-(\nu, \nu)$ must lie in $(p / 2) \mathbf{Z}=(3 / 2) \mathbf{Z}$.

But a direct calculation shows that $2\left(\lambda+\omega_{1}+\omega_{2}, \nu\right)-(\nu, \nu)=\frac{(q-1)(q+5)}{4}$. (The result [18, (6.2)] is a consequence of the strong linkage principle.)

Finally, for the case $G=G_{2}$ and $p \leq 3$, we see that $u$ has order 9 if $p=3$, and order 8 if $p=2$ (see Table 2). Then $\operatorname{dim} V \leq 9$, and again by [12, $V$ is a completely reducible $F G$-module unless $p=2$, and by dimensions we have that $V_{2}$ is a twist of the 7-dimensional indecomposable module considered in Lemma 2.9. But in this case Lemma 2.9 shows that $\left.u\right|_{V_{2}}$ is not regular. This completes the proof.

\section{The Case Where $H$ is Classical}

In this section we will establish Theorem 2.5 for the remaining classical groups.

Proposition 3.1. Theorem 2.5 is true if $H$ is classical.

Proof. Suppose first that $H$ is of type $B_{n}$, respectively $C_{n}$. Then a regular unipotent element of $H$ is regular in $D$, where $D=\operatorname{SL}(2 n+1, F)$, resp. $\operatorname{SL}(2 n, F)$. Therefore, by Proposition 2.11, $G$ is irreducible on $V$, and hence cannot be contained in a Levi subgroup of $H$.

Let $H$ be of type $D_{n}$ for $n>3$, and let $V$ be the natural $F H$-module. By Lemma 2.7 we may assume $\operatorname{char}(F)=p>0$. Let $u \in G$ be a regular unipotent element of $H$. By [16, Lemma 1.2], the Jordan normal form of $u$ on $V$ consists of two blocks with sizes $2 n-1,1$ if $p$ is odd, and $2 n-2,2$ if $p=2$.

Let $V^{u}$ be the fixed point space of $u$ on $V$. Obviously, $\operatorname{dim} V^{u}=2$. We deduce two auxiliary observations from this.

(i) If $X=X_{1} \oplus X_{2}$, where $X, X_{1}, X_{2}$ are $u$-stable subspaces of $V$, then the dimension of $X_{1}$ or $X_{2}$ is at most 1 if $p>2$, and at most 2 if $p=2$. (This follows by looking at $V^{u}$ and $V / V^{u}$.)

(ii) If $u^{p}=1$, then $p>5$. Indeed, if $p=2$, then we have $2 n-2 \leq 2$, which is a contradiction. If $p>2$, we have $2 n-1 \leq p$, which implies the claim as $n>3$.

We argue by contradiction and suppose that $G$ is contained in a proper parabolic subgroup of $H$. Then $G$ stabilizes a nonzero totally singular subspace of $V$. Let $W$ be a maximal $G$-stable totally singular subspace of $V, k=\operatorname{dim} W$, and let $P$ be the stabilizer of $W$ in $H$. Then $P$ is a parabolic subgroup of $H$ and $G \subset P$. 
Let $L$ be a Levi subgroup of $P$, so $L=\left(\mathrm{SL}(k, F) \times D_{n-k}\right) \cdot T_{H}$ if $n-k>1$, and $L=\mathrm{SL}(k, F) \cdot T_{H}$ if $n-k \leq 1$. Let $\pi: P \rightarrow L$ be the natural projection of $P$ onto $L$. By Lemma [2.6, $\pi(u)$ is a regular unipotent element of $L$. Then $\pi(u) \in[L, L]=\mathrm{SL}(k, F) \times D_{n-k}$ if $n-k>1$; otherwise $\pi(u) \in \mathrm{SL}(k, F)$. Denote by $\tau$ the further projection of $\pi(G)$ into $\operatorname{SL}(k, F)$. Then if $k>1, \tau(G)$ contains a regular unipotent element of $\mathrm{SL}(k, F)$ and so $\tau(G)$ is irreducible in $\mathrm{SL}(k, F)$ by Proposition 2.11, this is trivially true if $k=1$. Set $U:=W^{\perp} / W$. It is well-known that $W$ and $V / W^{\perp}$ are dual $G$-modules.

Suppose first that $U=0$. As mentioned above, $\tau(G)$ is irreducible on $W$; in particular, $\tau(G)$ belongs to the list of Lemma 2.10. For groups $G$ of type $A_{1}, C_{\ell}$, $B_{\ell}$ and $G_{2}$, all irreducible representations are self-dual [18, 1.8], so we have a selfextension here; however, every self-extension splits ([9, II.2.12(1)]), which means that $V$ is the direct sum of two $G$-submodules each of dimension equal to $(\operatorname{dim} V) / 2$, which contradicts the observation (i) above. For the remaining configuration of Lemma 2.10, $G=A_{\ell}$ with $\ell>1$. Then the highest weights of $G$ on $W$ and $V / W^{\perp}$ are $p^{i} \omega_{1}, p^{i} \omega_{\ell}$; then this extension splits by Lemma 2.2 and we once again have a contradiction.

We now have that $\operatorname{dim} U>0$, so $\operatorname{dim} U \geq 2$. We first show that $p>2$. Suppose $p=2$. Then the Jordan normal form of $u$ has a block $J_{2 n-2}$ of size $2 n-2$ on $V$, and $\tau(u)$ has a block (on $W$ ) of size at most $k \leq n-1$. This implies that $|u|>|\tau(u)|$, which is false. Thus, we assume until the end of the proof that $p>2$.

Suppose that $\operatorname{dim} U=2$. Then $G$ acts trivially on $U$, since $U$ is equipped with a nondegenerate $G$-invariant symmetric bilinear form; hence the restriction of $G$ to $W^{\perp}$ is an extension of $\tau$ by a trivial representation of $G$. We show that this extension splits. Indeed, if $G \cong A_{1}$, then $|u|=p$, and hence $\operatorname{dim} W \leq p$. By statement (ii) above, $p>5$ here. If $\operatorname{dim} W<p$, then the splitting follows from [12, Corollary 1.1.1]. If $\operatorname{dim} W=p$, then the highest weight of $\tau$ is $p^{j}(p-1) \omega_{1}$ for some integer $j \geq 0$, and we can use the linkage principle [7, 3.6]. The dominant weights linked in $A_{1}$ to 0 are of shape $i p-2$ for some integer $i>0$. This is not equal to $p^{j}(p-1)$ for $p>2$.

Continuing with the case $\operatorname{dim} U=2$, we must consider the groups of rank greater than 1. Note that $\operatorname{dim} W=k \leq \ell+1,2 \ell, 5,2 \ell+1,7,7$ in the cases (ii) - (vii), respectively, of Lemma 2.10. Then $\operatorname{dim} W^{\perp}=k+2 \leq \ell+3,2 \ell+2,7,2 \ell+3,9$, 9, respectively. By [12, Corollary 1.1.1], if $\operatorname{dim} W^{\perp} \leq \ell p$, then $W^{\perp}$ is a completely reducible $F G$-module, which contradicts (i) above. Therefore, we have only to deal with the cases where $\operatorname{dim} W^{\perp}>\ell p$. This yields the inequalities $\ell+3>\ell p$, $2 \ell+2>\ell p, 7>2 p, 2 \ell+3>\ell p, 9>2 p, 9>2 p$, respectively. Recalling that $p>2$, it follows that the possible configurations are when $p=3$ in the cases (iv) and (vii).

Consider the case (vii), when $p=3$. Then $k=7,|u|=9$, and hence $2 n-1 \leq 9$, which violates $k=7$ (since here we have $\operatorname{dim} V=2 \operatorname{dim} W+2=2 k+2$ ). Finally, suppose that $p=3$ in case (iv). Then $G$ is of type $C_{2}$ or $B_{2}$ (they are isomorphic). Then $W^{\perp}$ is completely reducible by [12, Theorem 1], giving a contradiction as above. (Recall that $W^{\perp}$ is an extension of $W$ by a trivial module and $W$ is as in Lemma 2.10(iv).)

We have now reduced to the case $\operatorname{dim} U>2$. Let $\sigma: G \rightarrow \mathrm{SO}(U)$ denote the representation of $G$ induced by $\phi$. Note that $\sigma \neq 1$ as $\sigma(G)$ contains a regular unipotent element of $D_{n-k}$. If $\operatorname{dim} U=4$, then $\mathrm{SO}(U)$ is a semisimple group of type $A_{1} A_{1}$, so $G \cong A_{1}$, and hence $p>5$ (by (ii) above) and $k \leq p$. As the Jordan 
form of $u$ on $V$ has a block of size $2 n-1$, it follows that $2 n-1 \leq p$. As $n=k+2$, we have $2 k+3 \leq p$, and hence $\operatorname{dim} W^{\perp}=k+4 \leq(p+5) / 2$. But since $p>5$, $(p+5) / 2 \leq p$, and [12, Corollary 1.1.1] implies that $W^{\perp}$ is a completely reducible $F G$-module, contradicting (i).

We consider one further special case, that is, when $\operatorname{dim} U=6$, and show that $W^{\perp}$ is a completely reducible $F G$-module. We have $D_{n-k}=D_{3} \cong A_{3}$; so $|u|=$ $|\sigma(u)|=p$, or $p=3$ and $|u|=9$. If $|u|=p$, then $p>5$ by (ii) above. In this case, $2 n-1 \leq p$ and $n=k+3$ imply that $\operatorname{dim} W^{\perp}=k+6 \leq \frac{p+7}{2} \leq p$, and so $W^{\perp}$ is a completely reducible $F G$-module as claimed. Thus we have $p=3$ and $|u|=9$. But then $2 n-1 \leq 9$ implies that $k \leq 2$. If $k=2$, then $|\tau(u)|=3$, and hence $|u|=3$, a contradiction. So $k=1$ and $\operatorname{dim} W^{\perp}=7$. As $D_{3} \cong A_{3}$, by Lemma 2.10. $G$ is of type $A_{3}$ or $C_{2}$. In the first case, [12, Corollary 1.1.1] implies that $W^{\perp}$ is completely reducible; hence we may asume $G=C_{2}$. Let $X$ be the 4 -dimensional natural module for $A_{3}$. Then the highest weight of $\left.X\right|_{G}$ is $3^{j} \omega_{1}$. It is well-known that $U$ is the wedge square of $X$ and that $G$ acts reducibly with composition factors of dimensions 5 and 1 on $\wedge^{2} X$. The highest weight of the nontrivial factor is $3^{i} \omega_{2}$. It follows that $U$ is completely reducible (as it is obviously self-dual). By [9, II.12.9, II.10.17(2)] and Lemma 2.2, $W^{\perp}$ is completely reducible again as claimed. Using the self-duality of $V$, in all cases, we have $\operatorname{dim} V^{u}>2$, which is a contradiction.

We now consider the remaining cases, where $\operatorname{dim} U \geq 8$. Then $\sigma(G)$ contains a regular unipotent element of $\mathrm{SO}(U) \cong D_{n-k}$. So the Jordan form of $\sigma(u)$ consists of blocks of size 1 and $2 n-2 k-1$. We show that $\sigma$ is an irreducible representation of $G$.

Indeed, suppose the contrary, that $\sigma(G)$ acts reducibly on $U$. By maximality of $W$, there is a proper $\sigma(G)$-invariant nondegenerate subspace $U^{\prime}$ of $U$ (recall that $p>2$ ), and hence $\sigma(G)$ stabilizes an orthogonal decomposition $U=U^{\prime} \oplus U^{\prime \prime}$. Considering the Jordan form of $\sigma(u)$, we may without loss of generality assume that $\operatorname{dim} U^{\prime \prime}=1$. Let $Z$ be the preimage of $U^{\prime \prime}$ under the mapping $W^{\perp} \rightarrow W^{\perp} / W$. Then $\operatorname{dim} Z=k+1$. We claim that $Z$ is a completely reducible $F G$-module. Indeed, if $|u|=p$, then $2 n-1 \leq p$ implies $k=\operatorname{dim} W \leq n-4 \leq \frac{p-7}{2}$, and the splitting follows from [12, Corollary 1.1.1]. If $|u|>p$, and so $G \neq A_{1}$, again by [12], we can assume that $\operatorname{dim} Z>p \ell$, equivalently, $\operatorname{dim} W>p \ell-1$. As above, the dimension of $W$ is at most $\ell+1,2 \ell, 5,2 \ell+1,7,7$ in the cases (ii) - (vii) of Lemma 2.10, respectively. As $p>2$, this is at most $p \ell-1$ unless $p=3$ and $G=G_{2}$, and then $|u| \leq 9$. As $2 n-1 \leq|u|$, it follows that $2 n=10$, and hence $\operatorname{dim} W=1$. Therefore, $Z$ is a reducible $F G$-module of dimension 2 , and hence trivial, so completely reducible as claimed. Now set $Z=W \oplus Z_{1}$, where $Z_{1}$ is a 1-dimensional, nondegenerate, $G$-invariant subspace. Then $G$ embeds in $\operatorname{SO}\left(Z_{1}^{\perp}\right)$, a simple group of type $B_{n-1}$. Moreover, the image of $G$ in this $B_{n-1}$ subgroup must contain a unipotent element of the $B_{n-1}$ with a Jordan block of size $2 n-1$ on $Z_{1}^{\perp}$, that is, a regular unipotent element. Since we have already established the result in case $H=B_{n-1}$, we see that the image of $G$ lies in no proper parabolic subgroup of $\operatorname{SO}\left(Z_{1}^{\perp}\right)$. But $W \subseteq Z_{1}^{\perp}$, hence a contradiction.

Thus, $\sigma$ is irreducible; so either $G=D_{n-k}$ or by [16. Theorem $\left.\mathrm{B}(\mathrm{iv})\right], G \cong A_{1}$, or $G \cong B_{3}$ and $\sigma$ is a Frobenius twist of the spinor representation of $G$. In the first case, when $G=D_{n-k}$, we see that $k=1$ since $\tau(G)$ must contain a regular unipotent element of $\mathrm{SL}(k, F)$ (see Lemma2.10). Now since $k=1$, we see that $\left.V\right|_{G}$ has precisely three composition factors, namely a twist of the natural module for 
TABLE 2. The maximal order of unipotent elements in the exceptional groups

\begin{tabular}{|c|l|l|l|l|l|l|l|l|l|l|}
\hline$p$ & 2 & 3 & 5 & 7 & 11 & 13 & 17 & 19 & 23 & 29 \\
\hline$E_{8}$ & $2^{5}$ & $3^{4}$ & $5^{3}$ & $7^{2}$ & $11^{2}$ & $13^{2}$ & $17^{2}$ & $19^{2}$ & $23^{2}$ & $29^{2}$ \\
\hline$E_{7}$ & $2^{5}$ & $3^{3}$ & $5^{2}$ & $7^{2}$ & $11^{2}$ & $13^{2}$ & $17^{2}$ & & & \\
\hline$E_{6}$ & $2^{4}$ & $3^{3}$ & $5^{2}$ & $7^{2}$ & $11^{2}$ & & & & & \\
\hline$F_{4}$ & $2^{4}$ & $3^{3}$ & $5^{2}$ & $7^{2}$ & $11^{2}$ & & & & & \\
\hline$G_{2}$ & $2^{3}$ & $3^{2}$ & $5^{2}$ & & & & & & & \\
\hline
\end{tabular}

$G$, and 2 trivial modules; but then applying Lemma 2.2 and [9, II.12.9, II.10.17(2)], we deduce that $V$ is a completely reducible $F G$-module contradicting $\operatorname{dim} V^{u}=2$. Now for the remaining two cases, observe that $\sigma$ is tensor indecomposable as $\sigma(u)$ is regular unipotent in $D_{n-k}$ (see [16, 1.5]). In addition, tensor indecomposable irreducible representations of $\mathrm{SL}(2, F)$ of even dimension are symplectic, which rules out the case with $G=A_{1}$. Therefore, $G$ is of type $B_{3}$, and $\operatorname{dim} U=8$. Note that the composition length of $W^{\perp}$ equals 2 , and the composition factors are given by $\tau$ and $\sigma$. By Lemma 2.10, $\tau$ is of highest weight 0 or $p^{i} \omega_{1}$ for some integer $i \geq 0$, and $\sigma$ is of highest weight $p^{j} \omega_{3}$. Then $W^{\perp}$ splits as $\sigma$ is faithful and $\tau$ is not faithful for $p>2$. Therefore, $W^{\perp}=W \oplus Y$, where $Y \cong U$ is a $G$-stable subspace of $W^{\perp}$. As above, this implies that $\operatorname{dim} V^{u}>2$, giving our final contradiction.

\section{The CASE WHERE $H$ IS EXCEPTIONAL}

In this section we will establish Theorem 2.5 in case $H$ is a simple algebraic group of exceptional type. By Lemma 2.7. we may assume $\operatorname{char}(F)=p>0$ and we let $o(H)$ be the maximum order of a unipotent element of $H$. This coincides with the order of a regular unipotent element of $H$. The value $o(H)$ is explicitly computed in 25]; we give these values when $o(H)$ exceeds $p$ in Table 2

We will rely heavily on [10, Theorem 1], where sufficient conditions for a semisimple subgroup of a simple exceptional algebraic group $H$ to be $H$-cr are given.

Proposition 4.1. Let $H$ be a simple algebraic group of exceptional type and $G$ a simple closed subgroup of $H$. If $G$ contains a regular unipotent element of $H$, then $G$ does not lie in any proper parabolic subgroup of $H$. (So Theorem 2.5 is true for $G$ of exceptional type.)

Proof. Arguing by contradiction, we suppose that $u \in G$, for $u$ a regular unipotent element of $H$, and $G \subset P$, a proper parabolic subgroup of $H$. As a proper Levi factor of $H$ cannot contain a regular element of $H$ (Lemma 2.6), $G$ does not lie in a Levi factor of $P$. Hence, we may use [10, Theorem 1] to reduce to a small number of possibilities, where Table 3 (taken from [10]) gives the maximal value $N(G, H)$ of the prime $p$ for which we must consider the pair $(G, H)$. If there is no value of $p$ in the column corresponding to $H$, then $G$ necessarily lies in a Levi factor of $H$ for all $p$. 
TABLE 3. $N(G, H)$

\begin{tabular}{|r|rrrrr|}
\hline & $H=G_{2}$ & $F_{4}$ & $E_{6}$ & $E_{7}$ & $E_{8}$ \\
\hline$G=A_{1}$ & 3 & 3 & 5 & 7 & 7 \\
\hline$A_{2}$ & & 3 & 3 & 5 & 5 \\
\hline$B_{2}$ & & 2 & 3 & 3 & 5 \\
\hline$G_{2}$ & & 2 & 3 & 7 & 7 \\
\hline$A_{3}$ & & & 2 & 2 & 2 \\
\hline$B_{3}$ & & 2 & 2 & 2 & 2 \\
\hline$C_{3}$ & & 2 & 2 & 2 & 2 \\
\hline$B_{4}, C_{4}, D_{4}$ & & & 2 & 2 & 2 \\
\hline
\end{tabular}

We now compare the above restrictions on $p$ with the information in Table 2 , where we give the orders of the regular unipotent elements in the exceptional groups. For $u \in H$ regular, $u \in G$ implies that the order of $u$ is at most the order of a regular unipotent element in $G$, as regular unipotent elements are dense in the variety of unipotent elements of $G$. If $G$ is of type $A_{n}$, the regular unipotent elements have order equal to the minimal power $p^{a}$ with $p^{a}>n$. The regular unipotent elements in $C_{n}$ are regular in $A_{2 n-1}$ (in the natural representation of $C_{n}$ ). As we mentioned in the proof of Proposition 3.1, the regular unipotent elements in $D_{n}$ acting on the natural $2 n$-dimensional representation space have exactly two Jordan blocks of sizes $(2 n-1,1)$ if $p \neq 2$, respectively, of sizes $(2 n-2,2)$ if $p=2$. So the regular unipotent elements in $D_{n}$ have order equal to the minimal $p^{a}$ with $p^{a} \geq 2 n-1$, respectively $p^{a} \geq 2 n-2$. Finally, we recall that the regular unipotent elements in $B_{n}$ are again regular in $D_{n+1}$ under the natural embedding of $B_{n}$ in $D_{n+1}$. Combining all of these results and comparing the orders, we see that $o(H)>o(G)$ unless $G$ is of type $G_{2}, p=5$ and $H$ is of type $E_{7}$.

We now consider this possibility in detail. Let $P$ be a proper parabolic subgroup of $H$ minimal with respect to containing $G$, and let $P=Q L$ be a Levi decomposition of $P$, where $Q=R_{u}(P)$. If $L^{\prime}$ has a simple factor of type $A_{k}$ for some $k$, then the minimality of $P$ implies that $G$ has a $(k+1)$-dimensional irreducible representation. If $L^{\prime}$ has a factor of type $D_{k}$ for some $k$, again the minimality of $P$ implies that there exists an irreducible $F G$-module of dimension $m$ for some $m \leq 2 k$, and on which $G$ stablizes a nondegenerate quadratic form. Given that $p=5$, we reduce therefore to the following configurations.

a) $L^{\prime}$ is of type $D_{4}, G$ stabilizes a nonsingular 1 -space of the natural module for $L^{\prime}$ and acts irreducibly on a nondegenerate complement to this space;

b) $L^{\prime}$ is of type $A_{6}$ and $G$ acts irreducibly on the natural module for $L^{\prime}$; or

c) $L^{\prime}$ is of type $E_{6}$.

In the first two cases, we will show that the semidirect product $G Q$ has a unique class of complements to $Q$, which implies that $G$ is conjugate to a subgroup of $L^{\prime}$, contradicting Lemma 2.6. The main tool here is [10, 1.7]. We refer to the table of highest weights of composition factors of $\left.Q\right|_{L^{\prime}}$ given in the proof of [10,3.4], as well as to [10, 2.10] for the restriction of these composition factors to the image of $G$ in $L^{\prime}$. Then for any such composition factor, say of highest weight $\mu$, we use the known information on the corresponding Weyl module $W(\mu)$ for $G$, when $p=5$, and we see that $\operatorname{Hom}_{F G}(\operatorname{rad}(W(\lambda), F)=0$. Then [10, 1.7] shows that there is indeed a unique class of complements to $Q$ in $G Q$. 
So it remains to consider the case $L^{\prime}$ of type $E_{6}$. By minimality of $P, \pi(G)$ is $L^{\prime}$-irreducible. Then [16, Theorem A] implies that $\pi(G) \subset M$, a maximal subgroup of type $F_{4}$. The Borel-Tits theorem [2] shows that $\pi(G)$ is $X$-irreducible in every intermediate subgroup $X \subset L^{\prime}$, so $\pi(G)$ lies in no proper parabolic subgroup of $M=F_{4}$. But then [16, Theorem A] provides a contradiction.

Proof of Theorem 1.2. As every unipotent element of $G$ is contained in the semisimple subgroup $[G, G]$, we can assume that $G$ is semisimple. Moreover, $[G, G]$ contains a regular unipotent element of $H$ if and only if the projection of $[G, G]$ into each simple factor of $[H, H]$ contains a regular unipotent element of the simple factor. So assume this is the case. By Proposition 2.3, each such projection is simple and by Theorem 2.5 lies in no proper parabolic subgroup of the simple factor, from which we deduce Theorem 1.2

\section{Proof of Theorem 1.4}

We can now apply the main theorem of 16 and Theorem 1.2 to determine the semisimple closed subgroups of $H$ which contain a regular unipotent element.

Let $G \subset H$ be a semisimple closed subgroup and suppose $u \in G$ for some regular unipotent element of $H$. Let $M \subset H$ be a maximal closed subgroup with $G \subseteq M$, necessarily of positive dimension. Then Theorem 1.2 implies that $M$ is not a proper parabolic subgroup of $H$, and so $M^{\circ}$ is reductive. Moreover, $u \in$ $G=G^{\circ} \subset M^{\circ}$ and by Proposition 2.3, we see that $\left[M^{\circ}, M^{\circ}\right]$ is in fact a simple group. Now, if $M^{\circ}$ is normalized by a maximal torus of $H$, then its root system corresponds to a subsystem of $\Phi(H)$ and therefore $M^{\circ}$ does not contain regular unipotent elements of $H$. In addition, we once again apply what is known about the Jordan block structure of regular unipotent elements in the classical groups. Then by [16, Theorem A, Theorem B], we deduce that one of the following holds:

(i) $M^{\circ}=A_{1}$ and $p>h$, the Coxeter number for $H$.

(ii) $M^{\circ}=F_{4} \subset E_{6}=H$.

(iii) $M^{\circ}=B_{\ell} \subset D_{\ell+1}=H$.

(iv) $M^{\circ}=C_{\ell} \subset A_{2 \ell-1}=H$.

(v) $M^{\circ}=B_{\ell} \subset A_{2 \ell}=H, p>2$.

(vi) $M^{\circ}=B_{3} \subset D_{4}=H$. (Here there are three conjugacy classes of such subgroups, interchanged by the graph automorphisms of $H$.)

(vii) $M^{\circ}=G_{2} \subset B_{3}=H, p>2$.

(viii) $M^{\circ}=G_{2} \subset C_{3}=H, p=2$.

(ix) $M^{\circ}=A_{2} \subset C_{4}=H, p=2$.

All of the above examples actually give rise to subgroups containing regular unipotent elements, except the example of (ix). Here $G$ acts irreducibly on $V$, the natural 8-dimensional module for $H$, and $\left.V\right|_{G}$ has highest weight $2^{j}\left(\omega_{1}+\omega_{2}\right)$, for some $j$. However, Lemma 2.10 implies that the regular unipotent elements in $G$ are not regular unipotent in $\mathrm{GL}(V)$ and hence are not regular in $H$.

We must now descend within each of the above configurations. So we choose a maximal positive-dimensional subgroup of $M^{\circ}$ arising as an example in the SaxlSeitz result. Then it is straightforward to see that this gives rise precisely to the list of Theorem 1.4. 


\section{ACKNOWLEDGEMENTS}

The authors are very grateful to I. Suprunenko and the anonymous referee for carefully reading the manuscript and for providing a detailed list of comments which allowed us to correct several inaccuracies in the first version. In addition, they thank Jean-Pierre Serre for his interest and for making some useful suggestions concerning the exposition.

\section{REFERENCES}

[1] A. Borel, Linear algebraic groups, 2nd edition, Springer-Verlag, New York, 1991. MR,1102012 (92d:20001)

[2] A. Borel and J. Tits, Eléments unipotents et sous-groupes paraboliques de groupes réductifs, Invent. Math. 12 (1971), 95-104. MR0294349(45:3419)

[3] N. Bourbaki, Groupes et Algèbres de Lie, Chaps. IV-VI, Hermann, Paris, 1968. MR 0240238 $(39: 1590)$

[4] R. Brauer and C. Nesbitt, On the modular characters of groups, Ann. of Math. (2) 42 (1941), 556-590. MR0004042 (2:309c)

[5] H. Enomoto, The characters of the finite Chevalley group $G_{2}(q), q=3^{f}$, Japan. J. Math. 2 (1976), 191-247. MR0437628 (55:10552)

[6] D. Gorenstein, R. Lyons and R. Solomon, The classification of finite simple groups, Vol. 3, Part I, Chapter A, Amer. Math. Soc. Providence, R.I., 1998. MR.1303592 (95m:20014)

[7] J. Humphreys, Modular representations of finite groups of Lie type, Cambridge Univ. Press, Cambridge, 2006. MR2199819 (2007f:20023)

[8] J.C. Jantzen, Low dimensional representations of reductive groups are semisimple, In: "Algebraic groups and related subjects: a volume in honor of R.W. Richardson" (eds. G. Lehrer et al.), Cambridge University Press, 1996. MR.1635685 (99g:20079)

[9] J.C. Jantzen, Representations of algebraic groups, Pure and Applied Mathematics, Vol. 131, Academic Press, Orlando, FL, 1987. MR899071 (89c:20001)

[10] M.W. Liebeck and G.M. Seitz, Reductive subgroups of exceptional algebraic groups, Memoirs Amer. Math. Soc., Vol. 580, Amer. Math. Soc., Providence, RI, 1996. MR1329942 (96i:20059)

[11] M.W. Liebeck and G.M. Seitz, The maximal subgroups of positive dimension in exceptional algebraic groups, Memoirs Amer. Math. Soc., Vol. 802, Amer. Math. Soc., Providence, RI, 2004. MR2044850 (2005b:20082)

[12] G.J. McNinch, Dimensional criteria for semisimplicity of representations, Proc. London Math. Soc. (3) 76 (1998), 95-149. MR1476899 (99b:20076)

[13] G.J. McNinch and D.M. Testerman, Nilpotent centralizers and Springer isomorphisms, J. of Pure and Applied Algebra 213 (2009), 1346-1363. MR2497582 (2010c:20059)

[14] R. Proud, J. Saxl, and D. Testerman, Subgroups of type $A_{1}$ containing a fixed unipotent element in an algebraic group, J. of Algebra 231 (2000), 53-66. MR.1779592 (2001f:20096)

[15] J. Saxl, Overgroups of special elements in simple algebraic groups and finite groups of Lie type, In: "Algebraic groups and their representations", NATO Adv. Sci. Inst. Ser. C Math. Phys. Sci., Vol. 517, Kluwer Acad. Publ., Dordrecht, 1998. MR1670776 (2000h:20087)

[16] J. Saxl and G.M. Seitz, Subgroups of algebraic groups containing regular unipotent elements, J. London Math. Soc. (2) 55 (1997), 370-386. MR1438641 (98m:20057)

[17] J.-P. Serre, Complète réductibilité. In: "Séminaire Bourbaki, 2003/2004", Astérisque 299 (2005), Exp. No. 932, viii, 195-217. MR2167207 (2006d:20084)

[18] G.M. Seitz, The maximal subgroups of classical algebraic groups, Memoirs Amer. Math. Soc., Vol. 365, Amer. Math. Soc., Providence, RI, 1987. MR888704 (88g:20092)

[19] G. M. Seitz, Maximal subgroups of exceptional algebraic groups, Memoirs Amer. Math. Soc., Vol. 441, Amer. Math. Soc., Providence, RI, 1991. MR.1048074 (91g:20038)

[20] T. Springer and R. Steinberg, Conjugacy classes, In: "Seminar on algebraic groups and related finite groups", Lecture Notes in Math., Vol. 131, Springer-Verlag, Berlin, 1970. MR0268192(42:3091)

[21] R. Steinberg, Lectures on Chevalley groups, Yale Univ., 1967. MR0466335 (57:6215)

[22] R. Steinberg, Regular elements of semisimple algebraic groups, Inst. Hautes Études Sci. Publ. Math. 25 (1965), 49-80. MR0180554(31:4788) 
[23] I.D. Suprunenko, Irreducible representations of simple algebraic groups containing matrices with big Jordan blocks, Proc. London Math. Soc. 71 (1995), 281-332. MR.1337469 (97a:20076)

[24] D. Testerman, Irreducible subgroups of exceptional algebraic groups, Memoirs Amer. Math. Soc., Vol. 390, Amer. Math. Soc., Providence, RI, 1988. MR961210 (90a:20082)

[25] D. Testerman, $A_{1}$-type overgroups of elements of order $p$ in semisimple algebraic groups and the associated finite groups, J. Algebra 177 (1995), 34-76. MR.1356359 (96j:20067)

École Polytechnique Federale de Lausanne, FSB-MathGeOM, Station 8, CH-1015 LAUSANNE, SWITZERLAND

E-mail address: donna.testerman@epfl.ch

Departimento di Matematica e Applicazioni, University of Milano-Bicocca, 53 via R. Cozzi, Milan, 20125, Italy

E-mail address: alexandre.zalesski@gmail.com 